\title{
Maintaining Intestinal Mucosal Integrity by Plugging Leaks with Homoectoine
}

\author{
Ricard Farré1,2 María Vicario ${ }^{2,3,4}$
}

Published online: 22 November 2018

๑) Springer Science+Business Media, LLC, part of Springer Nature 2018

The maintenance of a tight intestinal epithelial barrier is fundamental for the continuance of whole-body homeostasis. External (microbiota, alcohol consumption, diet) and internal (inflammation, stress, genetic predisposition) factors are associated with disruption of intestinal epithelial integrity and disease, though, despite intensive research, the specific mechanisms that alter intestinal barrier function remain unknown. Epithelial cells are linked by cellto-cell adhesion structures: The most apical structure is the tight junction (TJ), followed by the adherens junction and the desmosomes. In addition to its structural function, TJs also significantly contribute to the regulation of paracellular permeability. Most studies assessing paracellular permeability have described at least two populations of pores regulated by TJs: (a) a high-capacity charge-selective pore, permeable to small ions and small uncharged molecules (also referred as the "pore" pathway); and (b) a much larger low-capacity pore (also referred as the "leak" pathway) that is permeable to large ions and solutes regardless of charge. The pore pathway is regulated mainly by the claudin (CLDN) family of TJ proteins and the leak pathway by occludin (OCLN) and TJ proteins of the zonula occludens (ZO) family. The permeability of the leak pathway can be assessed by measuring the

Ricard Farré

ricard.farre@kuleuven.be

María Vicario

maria.vicario@vhir.org

1 Translational Research Center for Gastrointestinal Disorders, KU Leuven, Louvain, Belgium

2 Centro de Investigación Biomédica en Red de Enfermedades Hepáticas y Digestivas (CIBERehd, EHD16PI02), Barcelona, Spain

3 Laboratory of Translational Mucosal Immunology, Digestive Diseases Research Unit, Vall d'Hebron Institut de Recerca, Paseo Vall d'Hebron 119-129, 08035 Barcelona, Spain

4 Department of Gastroenterology, Hospital Universitari Vall d'Hebron, Universitat Autònoma de Barcelona, Paseo Vall d'Hebron 119-129, 08035 Barcelona, Spain transepithelial flux of small-medium size soluble molecules, including ethylene diamine tetraacetic acid (EDTA), mannitol, sucrose, inulin, and polyethylene glycols (PEG), or 4-20 kDa dextrans. In contrast, larger and most lipophilic molecules and intact bacteria do not cross the epithelium via the paracellular pathway; instead, they are either internalized via endocytosis and reach the basolateral pole via transcytosis and posterior exocytosis or enter into the lymphatics via the chylomicron pathway [1]. Altered epithelial barrier integrity, also known as increased intestinal permeability or 'leaky gut,' is associated with intestinal disorders such as celiac disease, inflammatory bowel disease (IBD), or functional gastrointestinal (GI) diseases (FGID) such as irritable bowel syndrome (IBS) and functional dyspepsia and in nonintestinal disorders such as diabetes, obesity, sepsis, Alzheimer disease, and multiple sclerosis. At least in intestinal disorders, the current hypothesis regarding the pathophysiological relevance of an altered epithelial barrier function is that luminal compounds (e.g., bacterial metabolites such as short-chain fatty acids) or bacterial structural components such as endotoxin, or food antigens can cross the 'leaky' epithelium, activating lamina propria immune cells, enteric and sensory nerves, or other structures expressing cognate receptors such as free fatty acid receptor (FFA) $2-3$ or Toll-like receptor 4 . In turn, pro-inflammatory cytokines released by immune cells or neural activation can exacerbate or further disrupt epithelial integrity. Inflammation as occurs in IBD and celiac disease can be severe or of a lesser magnitude as happens in FGID. Stress is also associated with gastrointestinal disease and, specifically, with altered intestinal permeability. FGIDs are characterized by higher levels of anxiety and depression; IBD relapse is also associated with stressful events. Basic and clinical studies have indicated that acute and chronic stress, whether physical or psychological, influences intestinal barrier function, including ion and water secretion, permeability, and mucus secretion. Other than direct effects on enterocytes or colonocytes, stress-induced intestinal permeability is also mediated via recruitment and 
activation of mononuclear immune cells such as mast cells, eosinophils, and macrophages that contribute to the local inflammatory response [2].

Several small molecules and peptides that act directly on epithelial cells such as glutamine, myosin light-chain kinase (MLCK) inhibitors, and zonulin peptide inhibitors have been tested in preclinical models of increased intestinal permeability and also in human diseases characterized by altered epithelial barrier function with different degrees of success. Ectoine (1,4,5,6-tetrahydro-2-methyl-4-pyrimidinecarboxylic acid) is a naturally occurring compound biosynthesized by several species of extremophilic microorganisms that confers resistance toward extreme salt concentrations and temperature stress. Ectoine was first identified in the microorganism Ectothiorhodospira halochloris, but is also present in diverse Gram-negative and Gram-positive bacteria. Currently, synthetic ectoine is used as an active ingredient in clinical skin care and sun protection products due to its beneficial properties such as stabilizing cellular components proteins, membranes, nucleic acids, as well as the contributing to whole cell homeostasis, protecting the skin for from damage due to ultraviolet irradiation and dryness. Homoectoine (4,5,6,7-tetrahydro-2-methyl-1H-(1,3)diazepine-4-carboxylic acid) derived from synthetic ectoine has biologic effects considered superior to ectoine.

In the present study [3], Castro-Ochoa et al. describe the protective effects of homoectoine on the epithelial barrier function during inflammation in a dextran sodium sulfate (DSS)-induced experimental colitis mouse model. The authors report a functional alteration of the leak pathway characterized by an increased permeability to Evans blue dye $(\mathrm{MW}=960.81 \mathrm{Da})$. Moreover, this alteration is associated at the molecular level by the downregulation of $\mathrm{ZO}-1$ and occludin expression. Interestingly, homoectoine not only reversed the "claudin switch" (downregulation of claudin-1 and upregulation of 'leaky' claudin-2) present in colitis but, more importantly, also restored the leak pathway by correcting the transepithelial passage of Evans blue and the expression of ZO-1 and occludin. This pathway is also altered in patients with FGID [4, 5] and understandably, in patients with IBD. All of these data indicate that from a molecular point of view, homoectoine has the potential to correct the increased permeability in humans by reversing the alterations of the pore pathway, but, more importantly, of the leak pathway. Unfortunately, and imperative for further potential therapeutic use, the authors did not measure the intestinal passage of larger molecules or bacteria despite published data consistent with increased transepithelial passage of intact bacteria such as E.coli and Salmonela typhimurium in clinical IBD [6] and IBS [7].

Remarkably, homoectoine not only restored epithelial barrier function but also reduced intestinal inflammation. At this moment, it is not known whether this amelioration is an indirect effect as a consequence of strengthening the epithelial barrier, or a direct effect mediated via inhibition of the pro-inflammatory NF- $\mathrm{B}$ pathway, a topic for further experimentation.

In conclusion, homoectoine is a very promising compound that could be used to reestablish intestinal epithelial integrity in inflammatory diseases and could also be used to prevent barrier dysfunction during infection, stress-associated disorders, or metabolic imbalance, subject to further experimental studies of diseases attributed to or associated with alterations of gut barrier function prior to clinical development.

\section{Compliance with ethical standards}

Conflict of interest Authors have nothing to disclose.

\section{References}

1. Farre R, Vicario M. Abnormal barrier function in gastrointestinal disorders. Handb Exp Pharmacol. 2017;239:193-217.

2. Rodino-Janeiro BK, Alonso-Cotoner C, Pigrau M, et al. Role of corticotropin-releasing factor in gastrointestinal permeability. $J$ Neurogastroenterol Motil. 2015;21:33-50.

3. Castro-Ochoa KF, Vargas-Robles H, Chanez-Paredes S, et al. Homoectoine protects against colitis by preventing a claudin switch in epithelial tight junctions. Dig Dis Sci. (Epub ahead of print). https://doi.org/10.1007/s10620-018-5309-8.

4. Vanheel H, Vicario M, Vanuytsel T, et al. Impaired duodenal mucosal integrity and low-grade inflammation in functional dyspepsia. Gut. 2014;63:262-271.

5. Martinez C, Vicario M, Ramos L, et al. The jejunum of diarrheapredominant irritable bowel syndrome shows molecular alterations in the tight junction signaling pathway that are associated with mucosal pathobiology and clinical manifestations. Am J Gastroenterol. 2012;107:736-746.

6. Keita AV, Salim SY, Jiang T, et al. Increased uptake of non-pathogenic $E$. coli via the follicle-associated epithelium in longstanding ileal Crohn's disease. J Pathol. 2008;215:135-144.

7. Bednarska O, Walter SA, Casado-Bedmar M, et al. Vasoactive intestinal polypeptide and mast cells regulate increased passage of colonic bacteria in patients with irritable bowel syndrome. Gastroenterology. 2017;153:948-960.e3. 